\title{
An Improved Design for Lab-used Thermostat with High Accuracy
}

\author{
Yu Yanting, Xu Yan, Xing Youguang \\ Shandong Provincial Key Laboratory of Ocean \\ Environment Monitoring Technology \\ Shandong Academy of Sciences Institute of \\ Oceanographic Instrumentation \\ Qingdao, China
}

\author{
Li Weidong \\ People's Hospital of Zhao Yuan \\ Yantai, China \\ yanting516@163.com
}

\begin{abstract}
This thermostat designed is for lab, with PID algorithm applied. The hardware includes PIC16F887 as processor, the 16-bit $\Sigma-\triangle$ A/D ADS1100 conversion as forward simulation, the thermal resistance PT100 as temperature sensor. The testing and control procedure is fixed by cooling and heating interaction.
\end{abstract}

Keywords-PIC16F887,XTR105, Temperature Control, high accuracy, $P I D$

\section{INTRODUCTION}

Temperature testing and control is closely related to our life, widely used in much fields (such as Industrial control, medicine and research organizations), which involves temperature control as parameters to ensure the work execution. Early thermostat system consists of relay temperature control circuit, it is concise on structure with low development pay and price. But it is low accuracy with more error due to much action on contact and bad contact quality [1][2].

The advanced thermostat is for industry testing and lab, which involves digital PID control algorithm, PIC16F887 as processor, 16-bit $\sum-\triangle$ A/D ADS1100 conversion chip, XTR105 as analogy front simulation, PT100 as temperature sensor and 4-bits LED as displaying. It completes temperature testing and control by cooling and heating interaction. The control range is $\quad-40^{\circ} \mathrm{C} \sim 20^{\circ} \mathrm{C}$, control accuracy $\pm 0.1{ }^{\circ} \mathrm{C}$. The software could be set based on requirements. It is with high accuracy, better stability and high speed in use.

\section{CONTENT}

\section{A. The overall design [3]}

1)Interface Graph

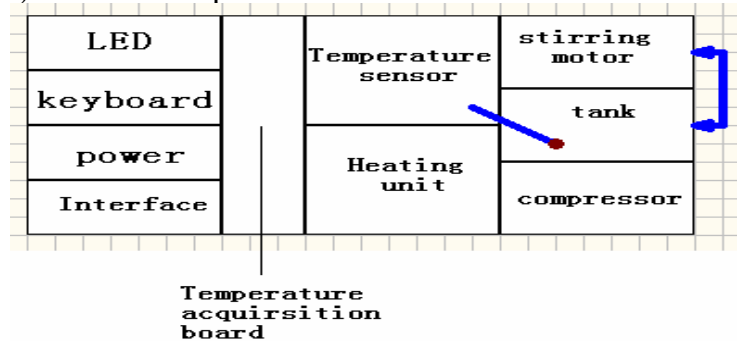

Figure 1. Interface Graph

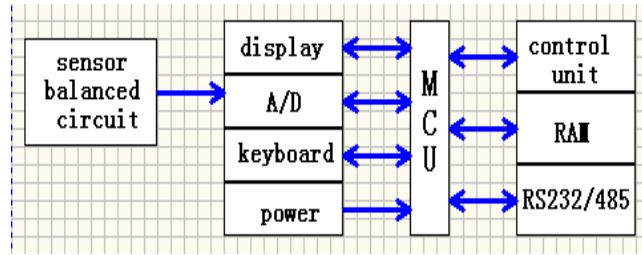

Figure 2. Temperature signal acquisition circuit Graph

2)Temperature signal acquisition circuit

Temperature sensor PT100 is used to test ethanol in tank, the signal is transferred into voltage signal through XTR105. The signal is transferred through processing such as amplifying, filter, A/D and be sent into PIC16F887 MCU system. It is compared with the default one in MCU system, if it meets the power-on point, the heater works following the digital PID control algorithm. This process could satisfy the higher accuracy on temperature control.

\section{B. Hardware design}

1)Center control and display unit

a) MCU selection [4]

We select PIC16F887 (Microchip) as the processor of data acquisition system. The architecture of PIC system is Harvard Bus, which apply the different bandwidth to realize "waterfall process" and "single-bit command", it is benefit on command executing efficient.

\section{b)Peripheral interface circuits}

(1)Communication interface

RS-232 is former, which could confirm closer link $(<15 \mathrm{M})$ and widely used in serial communication. Latest RS485 solves the previous shortcoming such as less distance and low rates. But an inconvenient DB9 transfer between RS-485 and RS-232 was injected in due to matching problem with PC.

c)keyboard circuit

This system includes 5 single-contact keys: SET(temperature configuration), RUN, UP(increase), DOWN(decrease) and RESET. Each key has special I/O. Some reuse realizes one key more function.

d)Display circuit

Because the thermostat is for lab and industry, a visible and clear vision is required. LED has more advantage than LCD, so we choose 4-bits, 0.8'LED.

2) The forward channel

a)Temperature sensor selection and interface [5] [6]

(1)Temperature sensor selection 
Sensor is a device which transfers income form into outcome form, it could support observation, measurement and research work. Platinum resistance sensor output $\mathrm{Pt}$ resistance under different temperature condition. It is fatal for PT resistance sensor testing to ensure the resistance all the time. Base on IEC751 international standard. The common producer is Pt100, with temperature parameter $\mathrm{TCR}=0.003851$. Its feature contains:

$$
\begin{aligned}
& \text { if }-200{ }^{\circ} \mathrm{C}<\mathrm{t}<0{ }^{\circ} \mathrm{C}, \\
& \mathrm{Rt}=\mathrm{R} 0[1+\mathrm{At}+\mathrm{Bt} 2+\mathrm{C}(\mathrm{t}-100) \mathrm{t} 3] \\
& \text { if } 0{ }^{\circ} \mathrm{C}<\mathrm{t}<850{ }^{\circ} \mathrm{C}, \\
& \mathrm{Rt}=\mathrm{R} 0(1+\mathrm{At}+\mathrm{Bt} 2)
\end{aligned}
$$

$\mathrm{Rt}$ is the resistance on $\mathrm{t}^{\circ} \mathrm{C}, \mathrm{R} 0$ is that on $0{ }^{\circ} \mathrm{C}$. The parameters are following:

$\mathrm{A}=3.90802 \times 10-3^{\circ} \mathrm{C}-1, \quad \mathrm{~B}=-0.5802 \times 10-7^{\circ} \mathrm{C}-2, \mathrm{C}=$ $-4.2735 \times 10-12^{\circ} \mathrm{C}-4$

(2)XTR105 circus[7] detail.

This section is described in chapter key technology in

\section{b)ADS1100 and interface circuit [8]}

ADS1100 is accuracy continuously self-calibration A/D. It has differential input and resolution to 16-bits.ADS1100 could make sampling on $8 / 16 / 32 / 128$ per second. The programmed gain amplifier (PGA) supply 8 times gain for weak signal measurement.

(1)Registers in ADS1100

ADS1100 has two major registers, both could be visited by $\mathrm{I} 2 \mathrm{C}$. Output register store the latest result; Configure register keep the function for user to configure the work of ADS1100 and check circuit state.

Output register: The latest $\mathrm{A} / \mathrm{D}$ result is stored in 16-bit output register, it is on complement form. It is clear and maintains " 0 " till the first A/D transfer from reset or power on.

Configure register: 8-bit register is for ADS1100 work, data rate and PGA configuration. The defaults configure register is $8 \mathrm{CH}$.

\section{(2)ADS1100 read operation}

The user is allowed to read from output register and configuration. It is needed to addressing and read 3 bits address. The front two bits represent content of output register and the last one is content of configuration register.

(3)ADS1100 write operation

The user is allowed to write new words in configuration register. It is needed to make writing operation on ADS1100 addressing to complete this function. One bit word is special for configuration register here.

3)Heating control circuit and cooling device

The core device is optocoupler Zero-triggered solid-state relays S203ZL, the input driving could be connected with TTL, and output controlling is $220 \mathrm{AC} / 50 \mathrm{~Hz}$. The minimum time interval is $10 \mathrm{~ms}$, current is $5 \mathrm{~A}$. We select resistance wire heater as load with $800 \mathrm{~W}$ power. We control compressor to refrigeration by the switch of system valve and balance valve.

4)Asynchronous serial communication circuit
RS-232 interface chip is MAX232 that keeps transferring of TIA/TIA-232-E and TTL/CMOS.

\section{System software design}

1)The system software design by C51

This system applies MPLAB IDE with HI-TECH PICC tool for system coding and compiling. It has more advantages.

2)system software composition

a)The flow chart of main program is in graph of appendix.

In flow loop, it is needed to type RESET key to reconfigure temperature point, the system will return to former mode and run then.

b) Keyboard module design

Keyboard includes 5 keys to fulfill following functions:

SET: enter temperature control interface and standby state;

RUN: enter temperature testing and control state;

UP: add 0.1 per type, continuous add on pressing;

DOWN: reduce 0.1 per type, continuous decrease on pressing;

RESET: reset system.

c)Display module design

PICs can afford high load driving capability through I/O. It can afford $25 \mathrm{~mA}$ on high and low level. The system utilizes above features, so we select 0.8 ' common cathode LED as display module.

d) temperature control circuit

The temperature signal is sent into PIC16F887 through XTR105, A/D. the MCU executes PID control of selfcalibration and output PMM signal. PID controls duty cycle of PWM in order to control compressor from relay switching.

e) The serial communication program of $M C U$.

Use USART of PIC16F887 to complete serial communication with PC.

$\mathrm{T} 1$ is rate generator, which is $9600 \mathrm{bps}$, working on autoreload mode. Its interface is 10-bit asynchronous receiver, it includes 8-bits for data group, 1-bit for start and 1-bit for end, two bits as one signal group, low value in the former and high in the post.

\section{KEY TECHNOLOGY}

\section{A. XTR105 circuit design}

The temperature testing circuit can be two-wire, threewire and four-wire. This system selects three-wire current source driven method to drive Pt resistance sensor. It could remove the resistance of $\mathrm{Pt}$ sensor, measure the change of sensor directly.

Illustration: RZ, RG, RLIN1 value calculation (assume temperature range $\mathrm{TMIN} \sim \mathrm{TMAX}$ )

$\mathrm{RZ}=\mathrm{RTD}$ resistance at TMIN

$\mathrm{RG}=[2(\mathrm{R} 2-\mathrm{RZ})(\mathrm{R} 1-\mathrm{RZ})] /(\mathrm{R} 2-\mathrm{R} 1)$

RLIN1 $=[$ RLIN $(\mathrm{R} 2-\mathrm{R} 1)] /[2(2 \mathrm{R} 1-\mathrm{R} 2-\mathrm{RZ})]$

$\mathrm{RLIN} 2=[(\mathrm{RLIN}+\mathrm{RG})(\mathrm{R} 2-\mathrm{R} 1)] /[2(2 \mathrm{R} 1-\mathrm{R} 2-\mathrm{RZ})]$

$\mathrm{R} 1=\mathrm{RTD}$ resistance at $(\mathrm{TMIN}+\mathrm{TMAX}) / 2$

$\mathrm{R} 2=\mathrm{RTD}$ resistance at TMAX 


$$
\mathrm{RLIN}=1 \mathrm{k} \Omega \quad(\text { Internal })
$$

B. Parameter calculation

$$
-10^{\circ} \mathrm{C}
$$$$
\mathrm{TMIN}=-40^{\circ} \mathrm{C}, \quad \mathrm{TMAX}=20^{\circ} \mathrm{C}, \quad(\mathrm{TMIN}+\mathrm{TMAX}) / 2=
$$

We can get following result based on $\mathrm{Pt}$ resistance formula:

$\mathrm{R}(\mathrm{TMIN}) \approx 84.27 \Omega, \mathrm{R}(\mathrm{TMAX}) \approx 107.79 \Omega$, $\mathrm{R}[(\mathrm{TMIN}+\mathrm{TMAX}) / 2] \approx 96.09 \Omega$

$\mathrm{R} 1=96.09 \Omega, \mathrm{R} 2=107.79 \Omega$

Based on three-wire connection RZ, RG, RLIN1 formula, we can get:

$\mathrm{RZ}=84.27 \Omega, \mathrm{RG}=47.52246 \Omega, \mathrm{RLIN} 1=49000 \Omega$, RLIN2 $=51066.72 \Omega$

RZ is made up of $100 \Omega$ precision wire wound potentiometer (turn to $84.27 \Omega$ ), $\mathrm{RG}$ is made up of $\pm 0.02 \%$ precision resistor, RLIN1 and RLIN2 are made up of $47 \mathrm{k} \Omega$ and $51 \mathrm{k} \Omega$ resistors.

The standard on equal testing is $-40^{\circ} \mathrm{C} \sim 20^{\circ} \mathrm{C}$ in temperature and $\pm 0.1^{\circ} \mathrm{C}$ in accuracy.

\section{INCUBATOR MEASUREMENT}

\section{A. Incubator measurement}

Connect the PT100 with measurement system and put it into incubator to calibrate. It is attention to the tightness of incubator to improve reliability, 10 times every 10 minutes. The results are in following table:

TABLE I. MEASURING RESULTS AND ERRORS OF THE CONSTANTTEMPERATURE-CHEST

\begin{tabular}{|c|c|c|c|}
\hline SetTemp $/{ }^{\circ} \mathrm{C}$ & Meausre $/{ }^{\circ} \mathrm{C}$ & Random Error & Sys error \\
\hline-40.00 & -39.92 & 0.01 & -0.08 \\
\hline-30.00 & -30.05 & 0.01 & 0.05 \\
\hline-20.00 & -19.97 & 0.01 & -0.03 \\
\hline-10.00 & -9.96 & 0.01 & -0.04 \\
\hline 0.00 & 0.01 & 0.01 & 0.00 \\
\hline 10.00 & 9.95 & 0.01 & -0.05 \\
\hline 20.00 & 20.00 & 0.01 & 0.00 \\
\hline
\end{tabular}

From these data the maximum random error is $0.05^{\circ} \mathrm{C}$, and get minimum on room temperature; The maximum error of measurement system is $-0.08{ }^{\circ} \mathrm{C}$, it could meet the requirement of high accuracy thermostat.

\section{B. Result analysis of the temperature control}

The volume of this thermostat is $0.6 \mathrm{~L}$. It is confirmed that it meets target in 60 s with over $1{ }^{\circ} \mathrm{C}$. It takes 360 s to get constant state completely.

\section{CONCLUSION}

The thermostat with three-wire constant current sourcedriven solves the influence from resistance of line and selfheating; the self-calibration of XTR105 realize the accuracy calibration for component drift and PT sensor. It improves the accuracy and reliability on testing; ADS1100 with differential input and 16-bit resolution conversion accuracy, it apply scaled-conversion power to maintain conversion accuracy and remove error; High accuracy testing mode with self-calibration incremental PID effectively control testing features and meet the requirements the user needs.

\section{ACKNOWLEDGMENT}

This paper was supported by the National Natural Science Foundation of China under Grant 40806044, and supported by the Qingdao Science and Technology Planning Project Fund of China under Grant 10-3-4-9-2-jch and supported by Research Fund for the Doctoral Program of the Shandong Academy of Sciences under Grant Y09-2.

\section{REFERENCES}

[1] YANG YAN-wei. A design of high-precision tem- perature measurement system[J]. Electronic Technology,2004(7):18-20(In Chinese).

[2] YANG Yong-jun. The actuality and development of the temperature measuring[J]. Metrology and Measur-ement Technology, 2009, 29(4):62-65(In Chinese)

[3] Jing-huai Zhang, Dong-an Yang. MCU water temperature control system $[\mathrm{J}]$. Journal of Beijing Electronic Science and Technolgy Institute, 1998(1): 22-23 (In Chinese).

[4] PIC16F887 Data Sheet 2008. www.microchip.com

[5] ZHU Yu-hong. An accurate measurement method for platinum resistance[J]. China Measurement Technology, 2007, 33(4):50-52(In Chinese).

[6] Rui-kun Gong, Jing-yuan Li. High accuracy PT resistence temperature measurement system implementation[J]. Instrumentation Technology, 2008(7): 9-10 (In Chinese).

[7] Burr-Brown Corporation Databook 1997. www.burrbrown.com/databook/XTR105.html

[8] ADS1100 Data Sheet 2008. http://focus.ti.com.cn

[9] Hong-cai Song. PID Controller Tuning[J]. China Metrology, 2003(11): 45 (In Chinese). 


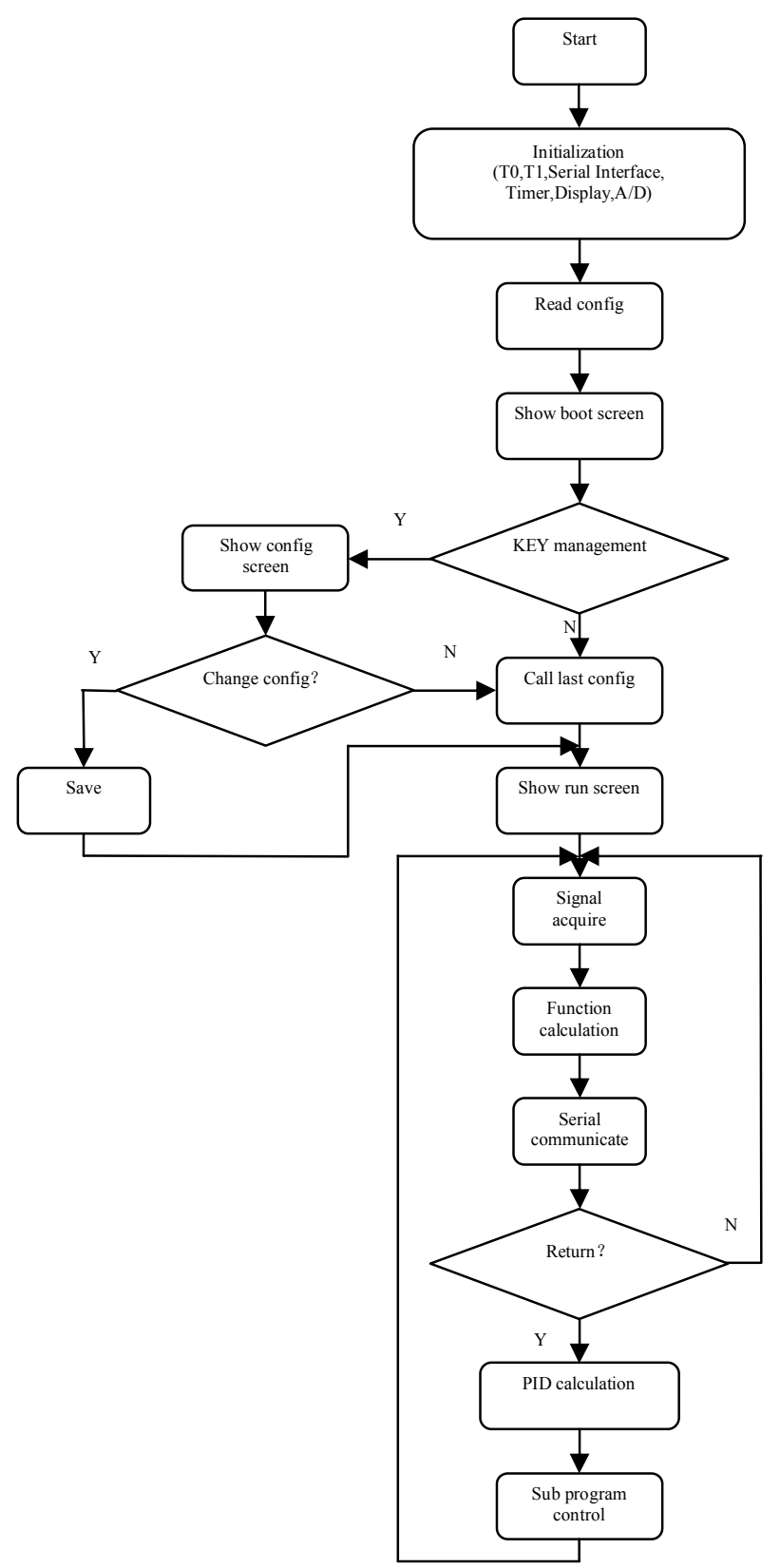

Figure 3. The main program flow chart 